\title{
YL-0919, a dual 5-HT 1 partial agonist and SSRI, produces antidepressant- and anxiolytic-like effects in rats subjected to chronic unpredictable stress
}

\author{
Yu-hua RAN ${ }^{1, \#}$, Xiao-xu HU ${ }^{1,2, \#}$, Yu-lu WANG ${ }^{1,3}$, Nan ZHAO ${ }^{1}$, Li-ming ZHANG ${ }^{1}$, Hua-xia LIU ${ }^{4, *}$, Yun-feng LI $\mathrm{LI}^{1, *}$ \\ ${ }^{1}$ Department of New Drug Evaluation, State Key Laboratory of Toxicology Medical Courtermeasures, Beijing Institute of Pharmacology \\ and Toxicology, Beijing 100850, China; ${ }^{2}$ Zoucheng Senior Vocational Technical School, Jining 273500 , China; ${ }^{3}$ College of Pharmacy, \\ Fujian University of Traditional Chinese Medicine, Fuzhou 350108, China; ${ }^{4}$ College of Nursing, Taishan Medical University, Taian \\ 271000, China
}

\begin{abstract}
YL-0919 has been identified as a novel dual 5- $\mathrm{HT}_{1 \mathrm{~A}}$ partial agonist and serotonin reuptake inhibitor. In the current study, we demonstrated that YL-0919 produced prominent antidepressant-like and anxiolytic-like effects in a chronic unpredictable stress (CUS) rat model. Male SD rats were exposed to CUS for 5 weeks; YL-0919 (1.25 and $2.5 \mathrm{mg} / \mathrm{kg}$ ) or a positive control fluoxetine (Flx, $10 \mathrm{mg} / \mathrm{kg}$ ) was orally administered daily. YL-0919 or Flx treatment significantly increased the sucrose preference rate, the locomotor activity in an open field test (OFT), the latency to feed in a novelty-suppressed feeding test (NSFT), and both the percentage of time spent in the open arms and the number of entries into the open arms in an elevated plus-maze test. YL-0919 or Flx treatment significantly suppressed the serum levels of ACTH and corticosterone in CUS-exposed rats. Additionally, YL-0919 or Flx treatment significantly enhanced the levels of cAMP, the expression of phosphorylated cAMP response element-binding protein (pCREB) and brain-derived neurotrophic factor (BDNF) in the hippocampus of CUS-exposed rats. Similar to FIx, YL-0919 treatment significantly enhanced the dendritic complexity, and increased the number of dendritic nodes as well as the spine length and number of branch nodes in the hippocampal pyramidal neurons of CUSexposed rats. Overall, our results reveal that YL-0919 suppresses the HPA axis and exerts antidepressant-like and anxiolytic-like effects in CUS-exposed rats, which are associated with the enhanced cAMP signaling and hippocampal dendritic complexity.
\end{abstract}

Keywords: YL-0919; antidepressants; anxiolytic agents; chronic unpredictable stress; HPA axis; hippocampus

Acta Pharmacologica Sinica (2018) 39: 12-23; doi: 10.1038/aps.2017.83; published online 31 Aug 2017

\section{Introduction}

Major depressive disorder (MDD) is a debilitating and recurring psychiatric disorder that affects over $15 \%$ of the American population and an increasing number of humans worldwide ${ }^{[1]}$. The World Health Organization website shows that depression is a common illness worldwide, with more than 300 million people being affected. Furthermore, the lifetime prevalence of MDD is $3.5 \%{ }^{[2]}$, and the 12 -month MDD prevalence is $2 \%$ in China $^{[3]}$. A series of antidepressants are available, including selective serotonin reuptake inhibitors (SSRIs) and serotonin norepinephrine reuptake inhibitors (SNRIs). However, only $50 \%-70 \%$ of patients exhibit mood improvements in response to initial antidepressant treatment ${ }^{[4]}$. Moreover, there is a time

\footnotetext{
\# These authors contributed equally to this work.

${ }^{*}$ To whom correspondence should be addressed.

E-mail huaxia66@163.com (Hua-xia LIU);

lyf619@aliyun.com (Yun-feng LI)

Received 2017-01-08 Accepted 2017-04-24
}

lag of weeks to months before currently available medications become effective. This time lag further highlights a major need not yet meet for novel rapid-acting and more efficacious antidepressant agents ${ }^{[5]}$.

Antidepressants that selectively target the 5-HT system may be effective in the management of different subtypes and symptoms of depression, suggesting that SSRIs may not be the only potential agents ${ }^{[6,7]}$. Given the complicated etiology and important roles of 5-HT and its receptors in antidepressant therapy, drugs combining the activities of $5-\mathrm{HT}_{1 \mathrm{~A}}$ receptor agonists with selective serotonin reuptake inhibitors may represent a new strategy for achieving antidepressants that have greater therapeutic efficacy. Approved in 2011 by the US Food and Drug Administration (FDA), vilazodone was the first antidepressant with dual 5- $\mathrm{HT}_{1 \mathrm{~A}}$ partial agonist and SSRI activity for the treatment of MDD. It is comparable to other known antidepressants and has fewer side effects ${ }^{[8]}$. Therefore, novel, ideal drugs that have a dual 5-HT reuptake blockade and 
a $5-\mathrm{HT}_{1 \mathrm{~A}}$ receptor partial agonist activity, which have been termed serotonin partial agonist-reuptake inhibitors (SPARIs), may hold promise for patients who cannot tolerate or have not responded to previous antidepressant monotherapies. Additionally, the use of SPARIs may extend to the treatment of other mental health conditions similar to those treated by serotonin selective reuptake inhibitors ${ }^{[9,10]}$. Chronic stress exposure also causes neuron atrophy in the prefrontal cortex and hippocampus regions in rodents ${ }^{[1,12]}$, and these effects might contribute to the decreased volumes of these regions that have been reported in brain imaging studies of MDD patients ${ }^{[13,14]}$.

A large number of clinical trials have suggested that stress can act as a precipitating factor in the onset of $\mathrm{MDD}^{[15]}$. The pathophysiology of depression and the neurobiology of stress are linked by their shared association with the hypothalamicpituitary-adrenal (HPA) axis ${ }^{[16,17]}$. To our knowledge, the relationship between the neuroendocrine effects of chronic stress and those of YL-0919 treatment is unknown.

Given all of the abovementioned factors, we designed and synthesized a series of compounds to search for SPARI compounds that have novel structure and activity to provide therapeutic treatment for depression and stress-related diseases. YL-0919 [(1-(1-benzyl-4-hydroxypiperidin-4-ylmethyl)$2(1 \mathrm{H})$-pyridinone hydrochloride] was identified as the final candidate due to its high combined selectivity as a serotonin reuptake inhibitor and $5-\mathrm{HT}_{1 \mathrm{~A}}$ receptor agonist and its strong antidepressant and anxiolytic-like effects. Chen et al demonstrated the therapeutic pharmacological properties of YL-0919 through an in vitro GTPYS binding functional assay and in vivo behavioral tests. The potency $\left(\mathrm{EC}_{50}\right)$ and efficacy $\left(E_{\max }\right)$ of YL-0919 in the rat hippocampus was determined to be $1.20 \pm 0.21 \mathrm{nmol} / \mathrm{L}$ and $85.11 \% \pm 9.70 \%$, respectively, by the $\left[{ }^{35} \mathrm{~S}\right]-\mathrm{GTP} \gamma \mathrm{S}$ binding assay. YL-0919 has high affinity for SERT/5- $\mathrm{HT}_{1 \mathrm{~A}}$, as the $K_{\mathrm{i}}$ was determined to be $0.72 \pm 0.10$ and $0.19 \pm 0.02 \mathrm{nmol} / \mathrm{L}$, respectively. In addition, it can potently inhibit 5-HT uptake in hSERT-transfected HEK293 cells and in the rat frontal cortex, with $\mathrm{IC}_{50}$ values of $1.93 \pm 0.18$ and $1.78 \pm 0.34 \mathrm{nmol} / \mathrm{L}$, respectively. When administered via the acute po route, a very low dose of YL-0919 could reduce the immobility time in the tail suspension and forced swimming tests in mice and rats ${ }^{[18]}$. In a recent study, the GTP $S$ binding assay was used in determining that YL-0919 is a 5-HT 1A $_{\text {A }}$ partial agonist $^{[19]}$. The molecular mechanisms underlying the antidepressant activity of YL-0919 are not clear and need further investigation.

Considering the relationship between molecular targets and therapeutic effects, the present study aimed to investigate the antidepressant-like effects of YL-0919 in vivo in a chronic unpredictable stress (CUS) model and the potential mechanisms of these effects related to dendritic complexity. It further aimed to determine whether chronic YL-0919 treatment could alleviate stress-induced behavioral abnormalities in the HPA axis. In addition, to further investigate the possible molecular mechanisms that may mediate the therapeutic effects of YL-0919, the study assessed the changes in dendritic complexity and the expression levels of brain-derived neuro- trophic factor (BDNF) protein and the portion of its upstream target cAMP response element-binding protein (CREB) that is activated [phosphorylated CREB (pCREB)] in the cortex.

\section{Materials and methods Animals}

Male Sprague-Dawley rats $(180 \pm 10 \mathrm{~g})$ were purchased from the Beijing Vital Laboratory Animal Technology Company (Beijing, China). The animals were maintained under standard conditions with controlled temperature $\left(23 \pm 1^{\circ} \mathrm{C}\right)$, humidity $(45 \%)$, and lighting $(12 \mathrm{~h} / \mathrm{d})$. The experiments were conducted according to the National Institutes of Health Guide for the Care and Use of Laboratory Animals (NIH Publications No.80-23, revised 1996). The experimental procedures were approved by the Institutional Committee on Animal Care and Use, and all efforts were made to minimize animal suffering and reduce the number of animals used for the experiments.

\section{Drugs and reagents}

Fluoxetine (Flx) was purchased from Sigma-Aldrich (St Louis, MO, USA). YL-0919 (purity $\geq 99 \%$ ) (Figure 1), which is approved for clinical research, was synthesized by the Department of Medicinal Chemistry at our institute. Rapid Golgi staining kits were purchased from FD NeuroTechnologies Inc (Washington DC, USA) (Table 1A). Unless otherwise stated, all compounds were dissolved in distilled water and administered to the rats via po in a volume of $2 \mathrm{~mL} / \mathrm{kg}$.

\section{CUS procedure}

The animals were exposed to a variable sequence of mild and unpredictable stressors for 5 weeks in a procedure that produces depressive-like behavioral changes ${ }^{[20,21]}$. The rats were randomly divided into the following five groups based on their sucrose preference in a final sucrose baseline test: control (nonstress), stress-vehicle (saline), stress-Flx $(10 \mathrm{mg} / \mathrm{kg})^{[22]}$, and stress-YL-0919 (1.25 and $2.5 \mathrm{mg} / \mathrm{kg})$. The vehicle or drugs were administered orally from 8:00 to 9:00 am $1 \mathrm{~h}$ prior to the stress procedure. Except for the control, non-stressed group, the animals were subjected to a total of eight different stressors (one stressor each day, see Table 1). The stressors included overnight illumination (19:00-7:00), white noise (110 dB) and strobe flash for $2 \mathrm{~h}$, cage tilting at $45^{\circ} \mathrm{C}$ for $24 \mathrm{~h}$, soiled cage for $24 \mathrm{~h}$, cold swimming at $10^{\circ} \mathrm{C}$ for $6 \mathrm{~min}$, tail pinch for $1 \mathrm{~min}$, water and food deprivation for $24 \mathrm{~h}$, and restraint stress for $1 \mathrm{~h}$. For details, see Table 1. Concomitant with CUS, drug treatments were given every morning and the sucrose preference test (SPT), open field test (OFT), and novelty-suppressed feeding test (NSFT) were performed after the drug treatments. An outline of the CUS and

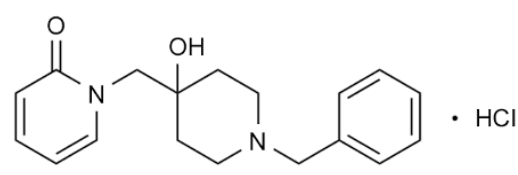

Figure 1. YL-0919 chemical structure. 
Table 1. The stressors used in the chronic unpredictable stress (CUS) procedures.

\begin{tabular}{ll}
\hline Type & Stressors \\
\hline 1 & Overnight illumination (19:00-7:00) \\
2 & White noise $(110 \mathrm{~dB})+$ strobe flash $2 \mathrm{~h}$ \\
3 & Cage tilting $45^{\circ} 24 \mathrm{~h}$ \\
4 & Soiled cage $24 \mathrm{~h}$ \\
5 & $10^{\circ} \mathrm{C}$ cold swimming 6 min \\
6 & Tail pinch 1 min \\
7 & Water deprivation+food deprivation $24 \mathrm{~h}$ \\
8 & Restraint stress $1 \mathrm{~h}$ \\
\hline
\end{tabular}

behavioral test design is presented in Figure 2 .

\section{Behavioral tests \\ SPT}

For the SPT on the 36th day of the CUS procedure, rats were exposed to a palatable sucrose solution (1\%, Sigma) for $48 \mathrm{~h}$, followed by $4 \mathrm{~h}$ of water deprivation and a $1 \mathrm{~h}$ exposure to two identical bottles: one filled with the sucrose solution and the other filled with water. This procedure was adapted from previous studies and has been previously used ${ }^{[23]}$. The sucrose and water consumptions were determined by measuring the volume of consumed liquid. Sucrose preference was defined as the ratio of the volume of consumed sucrose solution to that of the total volumes of consumed sucrose solution and water during the $1-\mathrm{h}$ test. Following the exposure to 5 weeks of stress, the sucrose preference test was again employed to evaluate the CUS model and drug actions. One hour before testing began, Flx $(10 \mathrm{mg} / \mathrm{kg})$ was administered via po and YL-0919 (1.25 and $2.5 \mathrm{mg} / \mathrm{kg}$ ) was administered orally.

\section{OFT}

The OFT began at 2:00 PM on the 37th day of the CUS procedure and drug treatment. The OFT was performed at the corner of a plastic $76 \times 76 \times 46 \mathrm{~cm}$ box (the floor was divided into nine equal sectors according to the method provided by Gray and Lalljee ${ }^{[24]}$ ). The rats were placed in the central grid and observed for $5 \mathrm{~min}$. The number of squares crossed with all paws (crossings) and the number of times the forepaws were raised (rearings) were recorded in the next 5-min session. The noise level in the room in which the experiment took place was minimal, and the open field box was cleaned before and after the test using $10 \%$ alcohol. One hour before testing began, Flx $(10 \mathrm{mg} / \mathrm{kg}$ ) was administered via po and YL-0919 (1.25 and 2.5 $\mathrm{mg} / \mathrm{kg}$ ) was administered orally.

\section{NSFT}

The novelty-suppressed feeding test (NSFT) was performed on the 38th day as previously described ${ }^{[25]}$. Before the test, the rats were food-deprived overnight. The rats were placed in an open field $(76 \mathrm{~cm} \times 76 \mathrm{~cm} \times 40 \mathrm{~cm}$ Plexiglas container) with a small amount of food in the center. The animals were allowed to explore the open field for $5 \mathrm{~min}$. The latency to feed, specifically the time required for the animal to approach and take the first bite of food, was recorded with a stopwatch. One hour before testing began, Flx $(10 \mathrm{mg} / \mathrm{kg})$ was administered via po and YL-0919 (1.25 and $2.5 \mathrm{mg} / \mathrm{kg})$ was administered orally.

\section{Elevated plus-maze test}

On the 39th day of the CUS procedure, the rats were placed in the center of a maze ( $40 \mathrm{~cm}$ above the floor) facing an enclosed arm. The numbers of entries (with all four paws) into the maze and the times spent in both the open and enclosed arms $(30 \times 5 \mathrm{~cm}$ and $30 \times 5 \times 15 \mathrm{~cm}$ high, respectively) were recorded for $5 \mathrm{~min}$ as previously described ${ }^{[26]}$. The percentages of entries in the arms and the time spent in the open arms were calculated, and these values were divided by the total arm entries and the total time, respectively. One hour before testing began, Flx $(10 \mathrm{mg} / \mathrm{kg})$ was administered via $p o$ and YL-0919 (1.25 and $2.5 \mathrm{mg} / \mathrm{kg}$ ) was administered orally.

\section{ACTH and corticosterone assays}

The rats were treated with antidepressants and/or saline for 5 weeks during the CUS procedure. To determine cytokine levels, serum was collected from their veins prior to death on the 40th day for the adrenocorticotropic hormone (ACTH) and corticosterone arrays. The samples were shipped on dry ice so that the protein concentrations could be assayed, and bead-based suspension protein arrays were analyzed at the measured levels of various cytokines as described. The sera samples were collected as usual. Next, enzyme-linked immunosorbent assay (ELISA) kits provided by the manufacturer (Abcam, Cambridge, UK) were used in measuring ACTH and

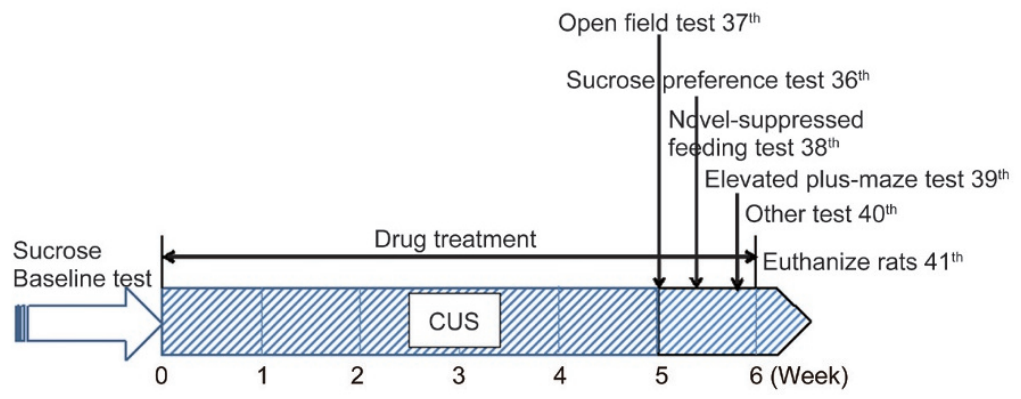

Figure 2. Outline of CUS procedure and behavioral tests experimental design. 
corticosterone (CORT), respectively. The standard curve for each array was first quantified with its respective kit, and the calibration was then exported and applied to the unknowns to obtain the calculated concentrations as described above.

\section{cAMP assays}

The punched cortex and hippocampus tissues were extracted with RIPA plus protease inhibitor combined with a phosphatase inhibitor cocktail and $0.5 \mathrm{mmol} / \mathrm{L}$ isobutyl-methylxanthine. The assays were performed with the Lance cAMP kit (PerkinElmer Lifesciences, Massachusetts, USA) according to the manufacturer's instructions. Comparison of the counts obtained at $665 \mathrm{~nm}$ with the cAMP standard curves allowed the quantitative determination of the cAMP concentrations. The signal at $615 \mathrm{~nm}$ is useful for identifying dispensing or quenching problems.

\section{Western blotting}

The rats were anesthetized by an overdose of 30\% urethane, and the brains were rapidly dissected and frozen at $-80^{\circ} \mathrm{C}$ until use. The frontal cortex, defined as the first $2 \mathrm{~mm}$ of cortex, was extracted by RIPA buffer with protease inhibitors and a phosphatase inhibitor cocktail (Thermo Scientific, Pierce, Rockford II, USA). Protein concentrations were measured using a BCA assay (BCA Protein Quantification Kit, Bitianyun Co, Ltd, Shanghai, China) according to the manufacturer's instructions. All samples were separated on $12 \%$ tricine gels (Invitrogen) and transferred to PVDF membranes. The membranes were blocked with 5\% nonfat dry milk in Tris-buffered saline $+0.1 \%$ Tween 20 (TBST) and incubated with primary antibodies diluted in milk overnight at $4^{\circ} \mathrm{C}$ (anti-BDNF IgG, anti-CREB IgG and anti-pCREB IgG, 1:500; anti- $\beta$-actin IgG, 1:2000; Cell Signaling Technologies) ${ }^{[27-30]}$. The membranes were washed in TBST and incubated with HRP-conjugated secondary antibodies for $1 \mathrm{~h}$ at $4^{\circ} \mathrm{C}$ (rabbit anti-goat, 1:5000; goat anti-rabbit, 1:5000). The specific bands were detected and analyzed using a Gel Pro Analyzer (Media Cybernetics, Bethesda, USA).

\section{Golgi staining test}

After the last behavioral tests (on the $40^{\text {th }}$ day of the CUS procedure), the rats were euthanized by injecting $30 \%$ urethane into their abdomen. Their brains were removed intact and rinsed with a $4 \%$ paraformaldehyde solution for less than 5 min. Golgi staining was performed according to the manufacturer's instructions with the FD Rapid Golgi Stain ${ }^{\mathrm{TM}}$ kit (Neuro Technologies, Ellicott City, MD, USA). In brief, the fresh brains were soaked in a solution containing potassium dichromate, mercuric chloride, and potassium chromate for 2 weeks at room temperature in the dark. Then, the tissues were transferred into a cyto-protectant solution and stored at $4^{\circ} \mathrm{C}$ for 3 days in the dark. Coronal sections of $100-\mu \mathrm{m}$ thickness from the dentate gyrus neurons (DG) of the hippocampus (PFC $\pm 0.25 \mathrm{~mm}$ from the coronal section through the injection site in the rostro-caudal direction, four to five sections per animal) were cut on a freezing microtome (Leica, Wetzlar, Ger- many) and collected on clean gelatin-coated microscope slides. After drying, the sections were re-hydrated in developing solution (an ammonium hydroxide-based solution) and dehydrated in a series of $50 \%, 75 \%, 95 \%$, and $100 \%$ ethanol washes. Finally, the sections were cleared with xylene and mounted on a coverslip with resinous mounting media. The neurons in the DG of the hippocampus were observed and photographed under a microscope. The dendritic length and branch counts of each nerve cell were measured with a digital planimeter ${ }^{[31]}$.

\section{Dendrite analysis}

The criteria applied to select neurons for reconstruction have been previously described ${ }^{[32,33]}$. In brief, after the behavioral experiments, the rats were executed with an injection of $30 \%$ urethane into their abdomens. In brief, only fully impregnated neurons located in regions without truncated dendrites were analyzed. The cells were digitally reconstructed and traced with Image-Pro Plus software (Media Cybernetics, Bethesda, MD, USA). Sholl analysis ${ }^{[34]}$ was used in determining the basal dendritic length and the number of dendritic nodes, which reflect dendritic complexity ${ }^{[35]}$. Equidistant (10 mm apart) concentric rings were placed over the dendritic tree tracings using the center of the soma as the reference point. The basal dendritic length was calculated by multiplying the number of intersections of each ring by $10 \mathrm{~mm}$, and the number of dendritic nodes was counted in each successive concentric zone. For quantitative analysis, four neurons from each hemisphere per section per animal and four animals per group were examined.

\section{Statistical analysis}

Significant differences between the treatment groups were identified by one-way ANOVA followed by the Bonferroni test. Differences were considered significant at the a priori level of $P<0.05$. The data were analyzed using GraphPad Prism (GraphPad Software Inc, San Diego, CA, USA).

\section{Results}

\section{Effects of YL-0919 on depressive-like CUS rats}

As illustrated in Figure 3A, chronic treatment with the SSRI antidepressant Flx (10 mg/kg, po, $P<0.01$ vs vehicle) and YL-0919 (1.25 and $2.5 \mathrm{mg} / \mathrm{kg}$, po) significantly increased the sucrose preference of rats that were subjected to the CUS procedure (Bonferroni test, $P<0.05$ vs vehicle for the $1.25 \mathrm{mg} / \mathrm{kg}$ dose and $P<0.01$ vs vehicle for the $2.5 \mathrm{mg} / \mathrm{kg}$ dose). As illustrated in Figure 3C and 3D, YL-0919 (1.25 and $2.5 \mathrm{mg} / \mathrm{kg}$ ) remarkably increased the number of rearings and crossings. Both YL-0919 and Flx remarkably increased the number of rearings (one-way ANOVA with Bonferroni test, $P<0.05$, $P<0.01$ vs vehicle for the $1.25 \mathrm{mg} / \mathrm{kg}$ and $2.5 \mathrm{mg} / \mathrm{kg}$ doses), which indicates their antidepressant-like effects.

\section{Effects of YL-0919 on anxiety-like CUS rats}

In the NSFT, which is widely used in assessing the effects of anti-anxiety agents, an acute administration of YL-0919 (1.25 and $2.5 \mathrm{mg} / \mathrm{kg}$ ) produced anxiolytic-like effects, as evidenced by the decreased latency to feed (one-way ANOVA with Bon- 
A

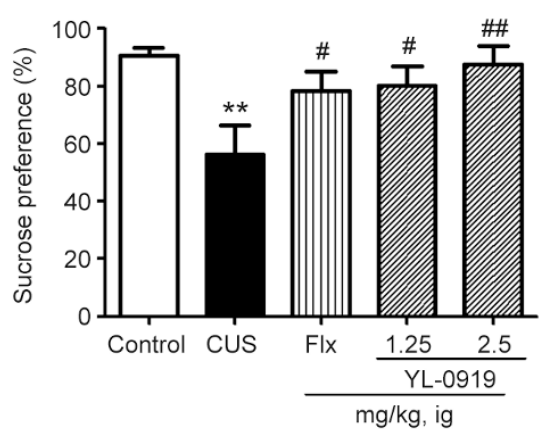

C

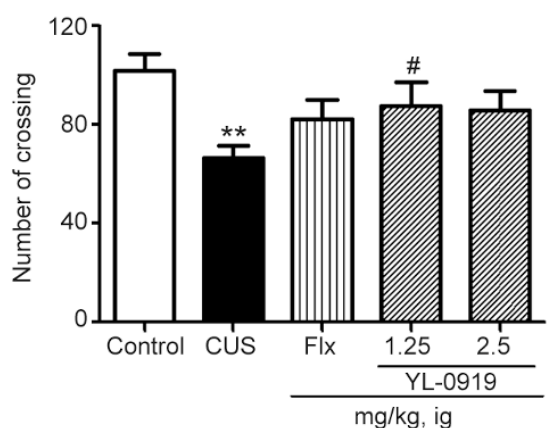

B

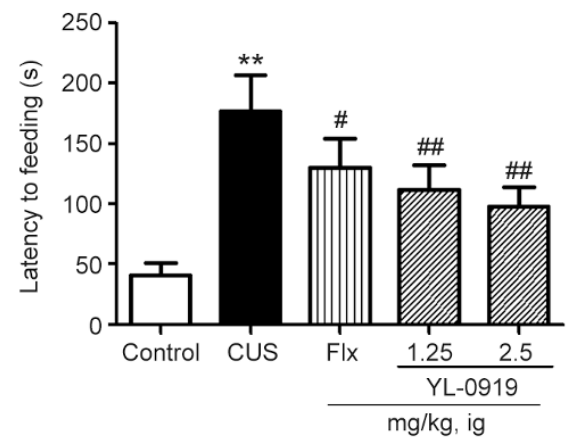

D

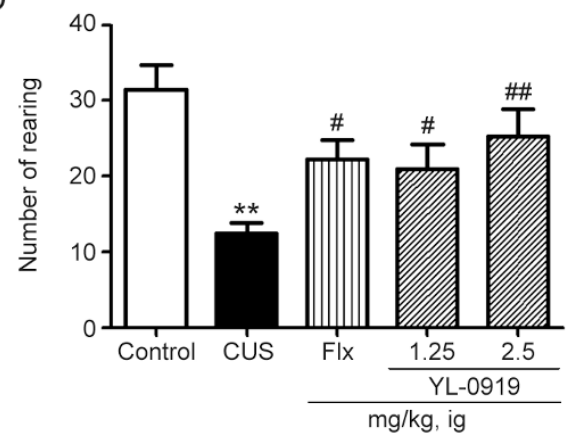

Figure 3. Effects of YL-0919 on depressive-like rats that were exposed to the CUS procedure. The rats were orally administered YL-0919 (1.25 and 2.5 $\mathrm{mg} / \mathrm{kg}$ ) or Flx (10 mg/kg) daily while being subjected to the CUS procedure for 5 weeks. The sucrose preference test was conducted on day 36 (A), and the NSFT was conducted after drug treatment (B). The effects of YL-0919 and Flx on the numbers of rat crossings (C) and rearings (D) were tested in the open field following the CUS procedure. The values represent the mean \pm SEM ( $n=8$ per group. ${ }^{* *} P<0.01$ vs control. ${ }^{\# \#} P<0.01$ vs CUS, analysis of variance with the Bonferroni test). Flx, fluoxetine; NSFT, novelty-suppressed feeding test; SPT, sucrose preference test.

ferroni test, $P<0.01$ vs vehicle for the 1.25 and $2.5 \mathrm{mg} / \mathrm{kg}$ doses; Figure 3B). A similar result was observed following Flx treatment $(10 \mathrm{mg} / \mathrm{kg}$, ip, $P<0.05$ vs vehicle).

The elevated plus-maze test is a well-established model for detecting both antidepressant- and anxiolytic-like behavior. In this test, the anxiolytic drug YL-0919 (1.25 and $2.5 \mathrm{mg} / \mathrm{kg})$, increased both the percentage of time spent in the open arms and the number of entries into the open arms. The results were significant at the dose of $2.5 \mathrm{mg} / \mathrm{kg}$ for the former and at the doses of 1.25 and $2.5 \mathrm{mg} / \mathrm{kg}$ for the latter compared with those corresponding with the vehicle. A similar effect was produced by Flx $(10 \mathrm{mg} / \mathrm{kg})$, which increased both the time spent in the open arms and the number of entries into the open arms, but only the change in the time spent in the open arms was significant $(P<0.05$ vs CUS). Neither YL-0919 nor Flx altered the total number of arm entries or the total time spent exploring the arms at the doses used (Figure 4A and 4B). Furthermore, in the hole-board test, each of the tested doses of YL-0919 and Flx significantly increased the time spent head dipping (Figure 4E). Together, these results indicate the potential anxiolytic effect of YL-0919.

\section{Effects of YL-0919 on the HPA axis in CUS rats}

Serum ACTH levels were remarkably increased in the CUSexposed group $(P<0.01)$ compared to those of the vehicle con- trol. Treatment with YL-0919 (1.25 and $2.5 \mathrm{mg} / \mathrm{kg}$ ) and Flx significantly reduced the levels of ACTH in these groups compared to those of the CUS-exposed group $(P<0.05)$ (Figure 5A). Corticosterone levels were found to be significantly increased $(P<0.01)$ in the rats exposed to CUS by itself, Flx, and YL-0919 $(1.25$ and $2.5 \mathrm{mg} / \mathrm{kg})$ could significantly reduce the corticosterone levels compared to those of the CUS groups $(P<0.05$, $P<0.01$, Figure 5B).

Effects of 0919 on the cAMP signaling pathway in depressivelike rats

The exposure of rats to CUS caused significantly lower levels of cAMP in the hippocampus (Figure 6A, $P<0.01$ vs vehicle control). Both YL-0919 and Flx caused an even further significant elevation of cAMP levels in the hippocampus $(P<0.01$ vs CUS). The cAMP content of the YL-0919 $(2.5 \mathrm{mg} / \mathrm{kg})$ and Flx groups showed remarkable enhancement compared to those of the CUS-exposed group ( $P<0.01$ vs CUS).

To assess the effects of YL-0919 and Flx on antidepressantrelated protein levels in the CUS-induced depressive rats, Western blotting analysis was performed. The protein expression level ratio of PCREB to CREB was significantly reduced by $52 \%$ in the CUS-exposed group, as shown in Figure 6B (i) $(P<0.01 v s$ control). The protein expression level ratio of pCREB to CREB was increased ( $P<0.01$ vs CUS) after both 
A

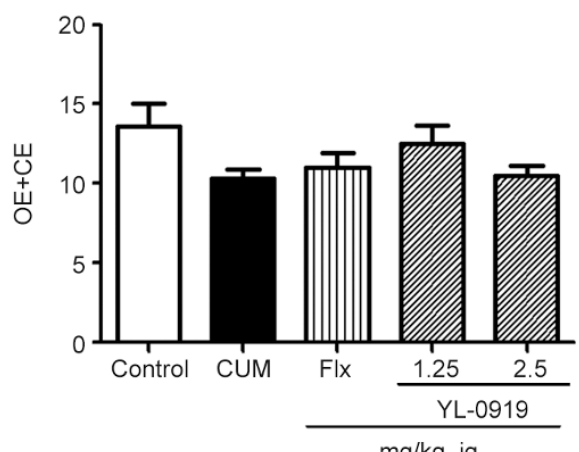

C

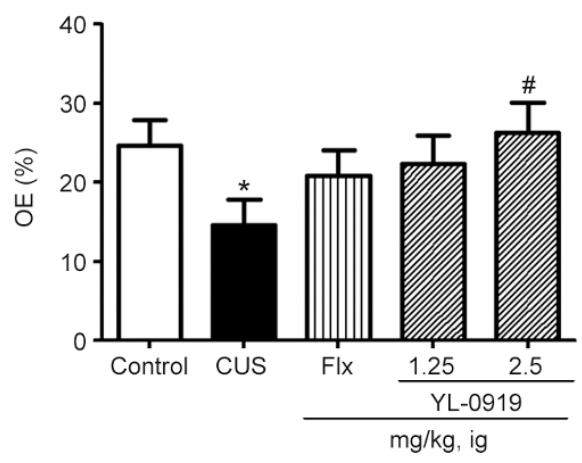

E

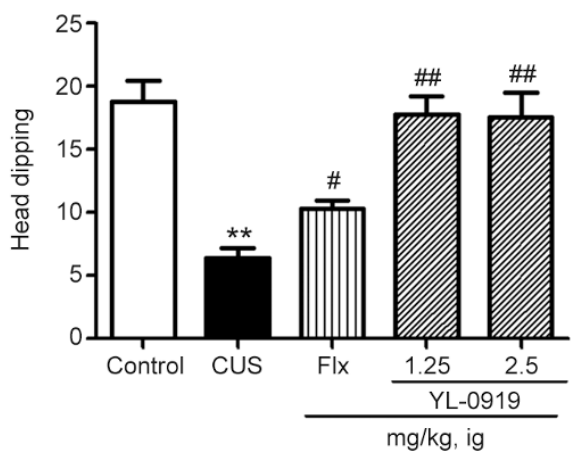

B

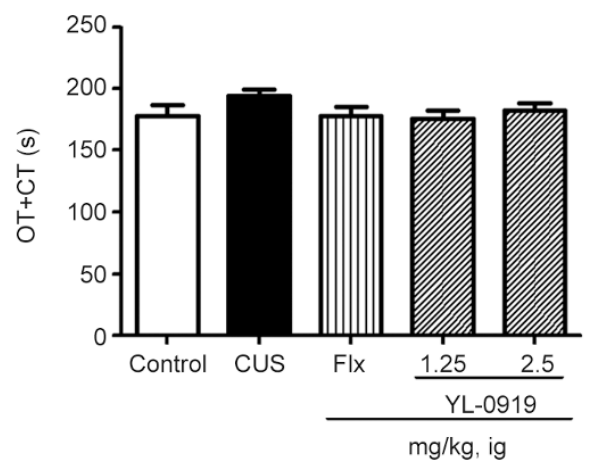

D

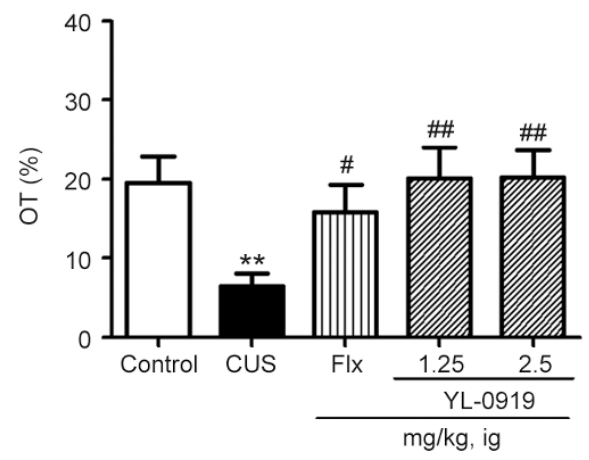

Figure 4. Effects of YL-0919 on the behavior of rats exposed to the CUS procedure in the elevated plus-maze test. Daily orally administration of YL0919 (1.25 or $2.5 \mathrm{mg} / \mathrm{kg}$ ) or Flx (10 mg/kg) were combined with the CUS procedure every day until execution. YL-0919 and Flx treatment increased the total time (A) and time spent in the open arms (B), the number of entries into the open arms (C), the amount of sustained time in the open arms (D), and the time spent head dipping (E) in the elevated plus-maze test. The values represent the mean \pm SEM $\left(n=8\right.$ per group. ${ }^{* *} P<0.01$ vs control.

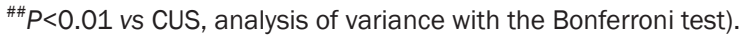

YL-0919 and Flx treatment (1.25 and $2.5 \mathrm{mg} / \mathrm{kg})$ by $154 \%$, $217 \%$, and $202 \%$, respectively, compared with that of the CUS group. The expression levels of BDNF are shown in Figure 6B (ii). The protein expression levels of BDNF were significantly lower in the CUS-exposed rats $(P<0.01$ vs control). The protein expression levels of BDNF were significantly higher in the YL-0919 group $(2.5 \mathrm{mg} / \mathrm{kg})(P<0.01$ vs CUS, respectively). In the Flx group, the BDNF expression level was remarkably increased compared with that of the CUS group $(P<0.01$ vs CUS).

\section{Effect of YL-0919 on dendrite formation in CUS rats}

The DG of the hippocampus region was microscopically observed following Golgi staining, and the images are shown in Figure 7A. The neurons were more dispersed in the depression model group than in the control group. In addition, the mean length of the dendrites was significantly shorter in the depression model group than in the control group, as illustrated in Figure 7B $(P<0.01)$. Both YL-0919 $(1.25$ and $2.5 \mathrm{mg} / \mathrm{kg})$ and Flx $(10 \mathrm{mg} / \mathrm{kg})$ remarkably increased the total lengths of the dendrites $(P<0.01)$. Regarding the total number of dendrite 


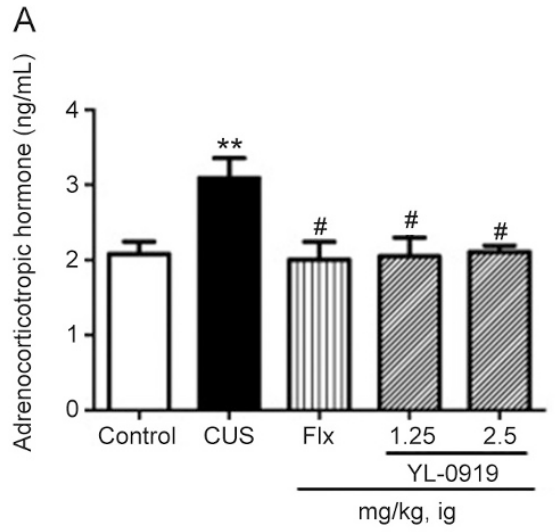

B

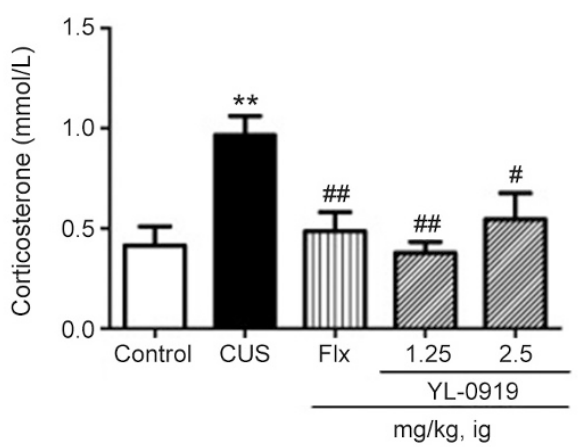

Figure 5. Effects of YL-0919 on ACTH (A) and corticosterone (B) levels in the serum of CUS-induced depressed rats. ${ }^{* *} P<0.01$ indicates significant differences between the control group and the CUS-induced group. ${ }^{\#} P<0.05$. ${ }^{\# \#} P<0.01$ indicates significant differences between the CUS-induced group and the Flx or YL-0919 (1.25 and $2.5 \mathrm{mg} / \mathrm{kg}$, ig) antidepressant drug treatment groups. Each value represents the mean $\pm S E M$. $n=8$.

A

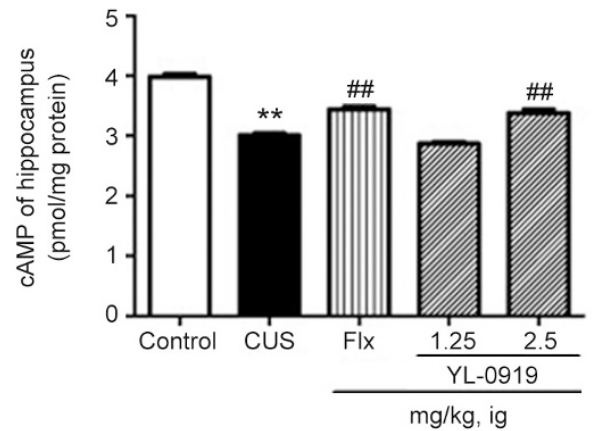

B
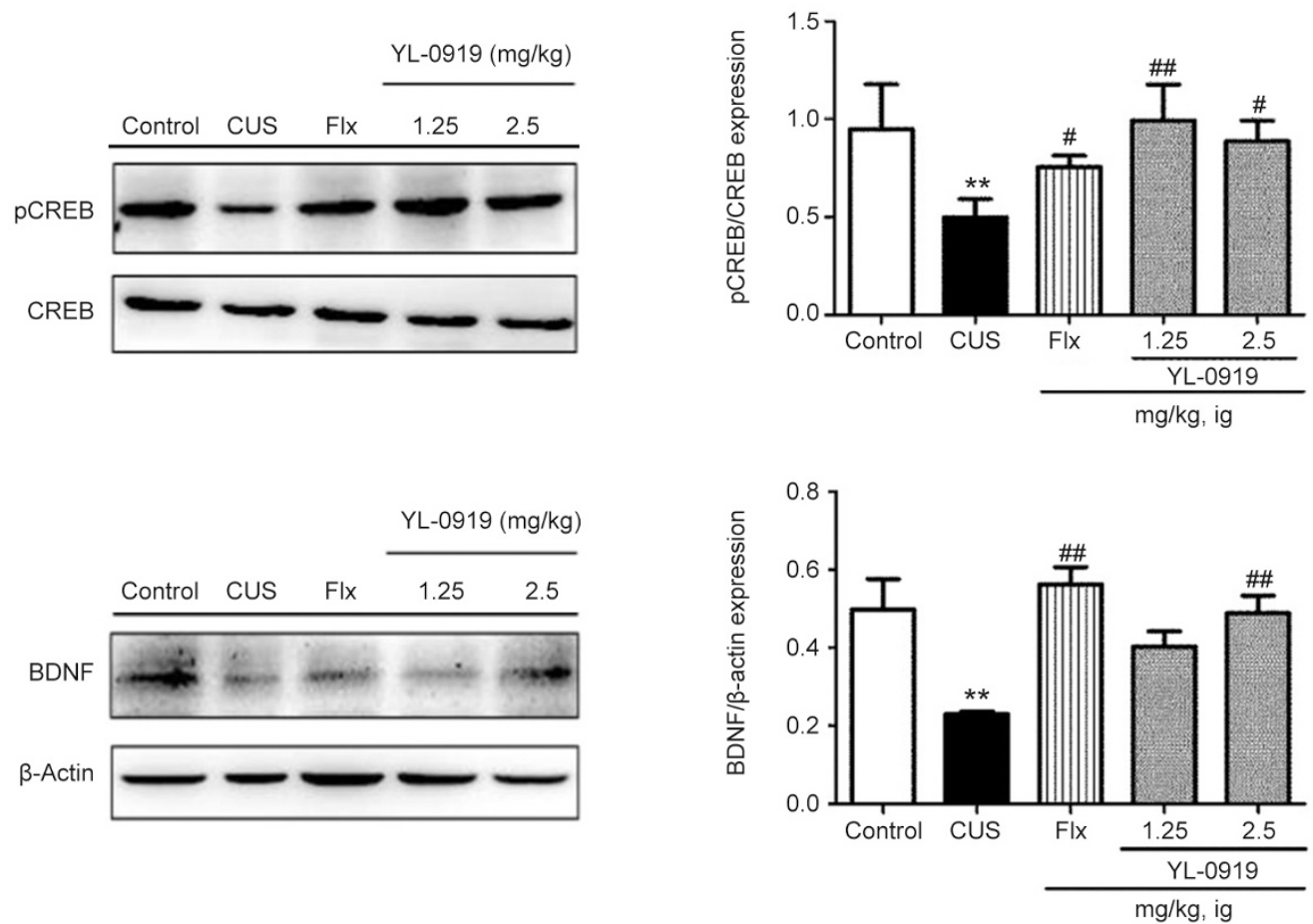

Figure 6. Effects of YL-0919 on the CAMP concentration (A) and protein expression (B) levels of pCREB/CREB and BDNF in the hippocampus of CUSinduced depressed rats, as determined by ELISA and Western blot analysis, respectively. ${ }^{* *} P<0.01$ indicates significant differences between the control group and the CUS-induced group. ${ }^{\#} P<0.01$ indicates significant differences between the CUS-induced group and the FIx or YL-0919 (1.25 and $2.5 \mathrm{mg} /$ $\mathrm{kg}$, ig) antidepressant drug treatment groups. Each value represents the mean \pm SEM. $n=8$ for ELISA and $n=4$ for Western blot. 
A

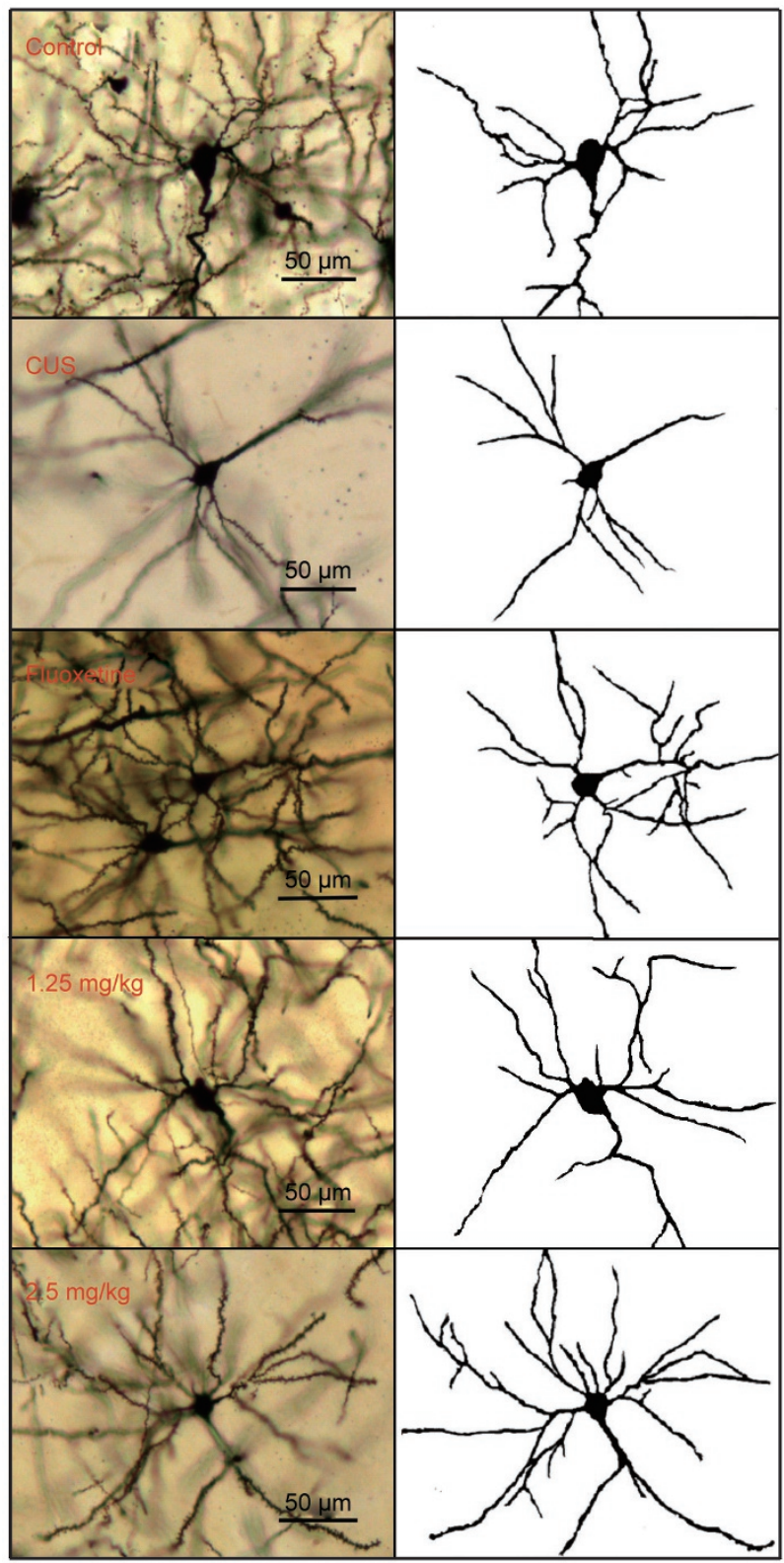

B

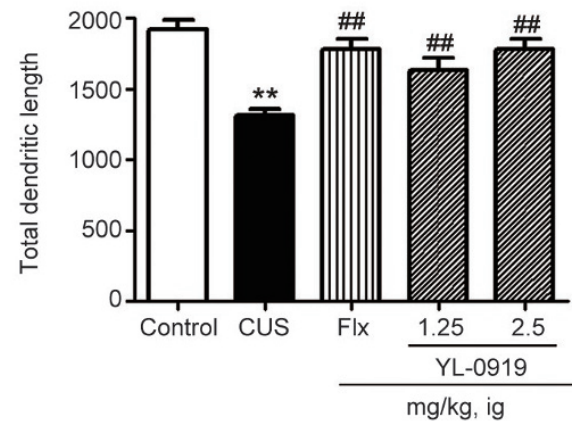

C

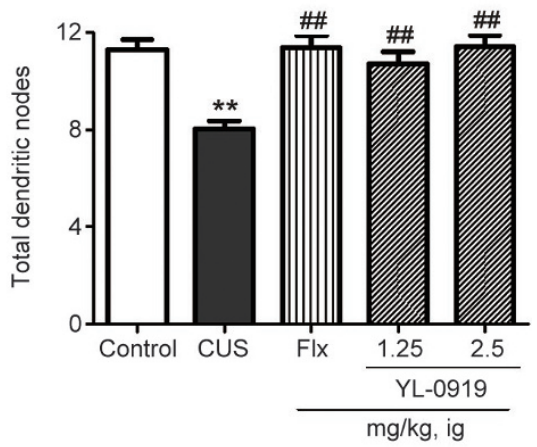

Figure 7. CUS exposure decreased the spine length and the number of DG branch nodes in the hippocampus of stressed rats, and these effects were reversed by chronic YL-0919 (1.25 and $2.5 \mathrm{mg} / \mathrm{kg}$ ) treatment (35 d). (A) Magnified (×20 objective) images of Golgi-impregnated hippocampus neurons. Scale bar=50 $\mu \mathrm{m}$. (B) Similar to the effects of chronic treatment with YL-0919 (1.25 and $2.5 \mathrm{mg} / \mathrm{kg})$, a significant increase in the total basal dendritic length was observed in rats treated with Flx (10 mg/kg). (C) YL-0919 (1.25 and $2.5 \mathrm{mg} / \mathrm{kg}$ ) and Flx (10 mg/kg) significantly increased the total dendritic nodes. Values shown represent the mean \pm SEM ( $n=96-120$ neurons from four animals per group. ${ }^{* *} P<0.01$ vs the control. ${ }^{\# \#} P<0.01$ vs the model, analysis of variance with the Bonferroni test).

nodes, comparable results were observed, as shown in Figure 7C $(P<0.01)$. These results indicate that YL-0919 enhances the formation of dendrites in terms of length and number.

\section{Discussion}

In the present study, our results demonstrate that YL-0919 exerts antidepressant-like and anxiolytic-like effects on behav- ior and suppresses the HPA axis, which is involved in the enhancement of cAMP signaling stability and hippocampal dendritic complexity.

The CUS model is most popularly used for pre-clinical evaluations of antidepressants. Behavioral deficits and neurochemical or neuroendocrine disturbances induced by CUS stimuli are extremely similar to the symptoms or alterations 
observed in depressive patients in the linic $^{[23,36]}$. In our study, 5 weeks of stress induced significant reductions in the main symptoms that indicate major depression (i.e., locomotor activity and sucrose preference), which coincides with a lack of reactivity and anhedonia. The 5-week design CUS paradigm is an integrated procedural animal model of depression with good predictive validity (behavioral alterations are reversed by chronic treatment with most current antidepressants), face validity (almost all depression symptoms are demonstrated), and construct validity (CUS causes anhedonia, the core symptom of depression $)^{[37,38]}$. Anhedonia is defined as "the decreased capacity to experience pleasure of any sort ${ }^{\prime \prime}{ }^{[39]}$. To elucidate the effects of YL-0919 on the depressed animals, we adopted the CUS model in rats. This 5-week design offered a closer resemblance to the chronically stressful life events that are major risk factors for developing depression ${ }^{[40]}$. The effectiveness of this procedure was monitored by the decrease in the responsiveness to rewards (sucrose preference). The effectiveness was also confirmed by the elevated levels of corticosterone in the serum, the degradation of the physical coat state, and behavioral deficits identified with the novel suppressed food test and open field test. These alterations were consistent with previous studies ${ }^{[41]}$. Even though there are numerous antidepressants available, only one third of patients show significant mood improvement in response to initial antidepressant therapy ${ }^{[14]}$. Moreover, there is a lag time of weeks to months with the medications currently available ${ }^{[42]}$. Preclinical and clinical studies have determined that ketamine can produces rapid antidepressant response (within hours) in treatment-resistant MDD patients ${ }^{[43,44]}$. Single administrations of other SSRIs, such as fluoxetine, or other antidepressant cannot take effect in clinical trials. Therefore, a chronic concomitant drug was used in evaluating the antidepressant effects of these kinds of drugs. Concomitant YL-0919 administration restored these behavioral deficits, suggesting its antidepressant-like activity. Furthermore, the CUS model has also been reported to induce anxiety-like symptoms ${ }^{[45,46]}$. The NSF test was initially applied to examine the effects of anti-anxiety reagents ${ }^{[37]}$. Chronic treatment with antidepressants significantly reduces the initial latency with which animals begin to eat in a novel environment. The doses used in this study were consistent with those used in other studies and within the effective dose ranges. In this study, YL-0919 significantly increased the sucrose preference rates of the CUS rats at the applied doses $(1.25,2.5 \mathrm{mg} / \mathrm{kg})$. In the NSFT, increasing the administered dose of YL-0919 remarkably decreased the latency to feed. The elevated plus-maze test was applied to further investigate the anxiolytic effects of YL-0919. In this model, YL-0919 increased both the percentage of entries and the time spent in the open arms, which suggests that YL-0919 possesses anxiolytic activity. Furthermore, at the same dose, YL-0919 did not influence the total arm entries and time spent. The findings from this test are consistent with those of a previous report that demonstrated that YL-0919 elicits antidepressant effects in various animal models. A 5-week repeated treatment with WAY100635, which blocks the 5- $\mathrm{HT}_{1 \mathrm{~A}}$ receptor, reduced anxiolytic-like effects in mice in the elevated plus-maze test ${ }^{[18]}$. Compared with YL-0919, Flx elicited comparable results and did not affect the total arm entries. Taken together, the in vivo results from this study indicate that the antidepressant and anxiolytic effects of YL-0919 and Flx are equivalent at the doses tested.

Extended impairment of HPA axis feedback inhibition has been reported in both preclinical and clinical studies ${ }^{[47,48]}$. In our study, we found that rats subjected to CUS showed a depressive-like dysfunction of HPA axis feedback, which was associated with an increase in spontaneous corticosterone secretion. Our findings showed that chronic YL-0919 administration could also alleviate stress-associated physiological changes in ACTH and corticosterone. Elevated corticosterone (in rodents) levels is one of a hallmark of HPA axis feedback inhibition ${ }^{[49,50]}$. The hippocampus contains an exceptionally high-level exression of corticosteroid receptors, which are involved in negative feedback inhibition of the HPA axis in learning and memory processes ${ }^{[51]}$. Our findings provide evidence that the antidepressant effects of YL-0919 may be the result of normalization of the HPA axis. Rat behavioral deficits are associated with HPA axis feedback dysfunction, which is characterized by an exaggerated corticosterone response ${ }^{[52]}$. Our results showed that YL-0919 could reverse stress-induced changes in behavior, physiology, and expression of proteins involved in HPA axis control.

In animal models of chronic stress, neurons in the hippocampus and the frontal cortex display atrophy and downregulated BDNF expression in response to repeated stress ${ }^{[40]}$. The BDNF gene contains a cAMP response element (CRE), which $\mathrm{pCREB}$ binds and thereby enhances transcription. Clinical observations suggest that glucocorticoids may interfere indirectly with this process when the cortisol-GR complex is bound to CREB because its phosphorylation is prevented and thus CRE-regulated BDNF expression is blocked. The chronic administration of various antidepressants increases the expression, phosphorylation, and function of CREB and its downstream target gene BDNF in the rat hippocampus and other limbic brain regions thought to be involved in depression ${ }^{[53,54]}$. In accordance with this view, we found in the present study that chronic (5 week) YL-0919 treatment combined with CUS ameliorated the reduction of BDNF and PCREB/CREB levels that were produced by the stress protocol. Moreover, these findings raise the possibility that YL-0919 may reverse or protect hippocampal and cortical neurons from further damage in response to CUS, by up-regulating PCREB and BDNF. These findings shed light on the mechanism by which YL-0919 acts at the psycho-neuroendocrine level, particularly as a modulator of the HPA axis.

The behavioral results indicate the effect of YL-0919, and comparable results were observed in the Golgi staining test. In the Golgi staining test, both the numbers and lengths of dendrite complexity were significantly increased at the YL-0919 doses of 1.25 and $2.5 \mathrm{mg} / \mathrm{kg}$. These findings indicate that YL-0919 at the effective doses has a similar influence on both behavior and the synapse in terms of dendritic formation. 
Neuron atrophy in the rodent prefrontal cortex and hippocampus can be induced by CUS exposure ${ }^{[11,12,55,56]}$. Brain imaging of MDD patients has revealed that these effects could contribute to decreased volume of these regions ${ }^{[9,10,57]}$. Synaptic morphology and structural plasticity are the material bases of functional synaptic plasticity. Neuron atrophy results in fewer dendritic spines and a decreased proportion of larger synapses compared with those of normal controls ${ }^{[58]}$. YL-0919 at its effective doses and Flx elicited increases in the numbers of dendrite nodes. Additionally, both YL-0919 and Flx increased the dendritic lengths, as indicated by Golgi staining. These results suggest that YL-0919 can enhance dendritic complexity. These alterations in basal synaptic morphology are correlated with enhanced mean synaptic strength and impaired synaptic modifiability in the cortex ${ }^{[59]}$. Our present findings indicate that chronic YL-0919 treatment increases the dendritic complexity and implicates potential similar pathways for synaptic plasticity. Therefore, YL-0919 may reverse depressivelike behavior and dysfunction due to increasing the number of dendrite nodes and their length and enhancing synaptic activity. In other words, the amelioration of dendrite complexity by YL-0919 influences the function of the neuronal system and results in behavioral changes.

In conclusion, this study clearly demonstrates an antidepressant-like effect of YL-0919, which is produced by ameliorating HPA axis dysfunction and improving the behavioral deficits induced by CUS. Furthermore, the effects of YL-0919 on the behavioral deficits induced by CUS may be related to the enhancement of dendritic complexity in terms of both node number and dendritic length. Moreover, the data presented herein suggest that the PCREB/CREB and BDNF protein levels in the brain are increased, specifically in the hippocampus. These findings support the potential therapeutic application of YL-0919 as an antidepressant. The methods presented herein were limited due to the ethological and biochemical parameters of the CUS model used in this study. Other animal models of depression and specific inhibitors that target the main regulated molecules should be utilized and verified through diverse methods. Further studies are currently being conducted in our laboratory to confirm whether and how the antidepressant effects of YL-0919 are related to synaptic plasticity, as well as to determine the molecular mechanisms of the HPA axis. Molecular biochemical methodology is being employed to take advantage of the pros and cons of knock-in and knockdown in vitro and in vivo models.

\section{Abbreviations}

MDD, major depressive disease; Flx, fluoxetine; TCAS, tricyclic antidepressant; MAOIs, mono-amine oxidase inhibitor; SPARI, serotonin partial agonist-reuptake inhibitor; CUS, chronic unpredictable stress; SPT, sucrose preference test; OPT, open field test; NSFT, novelty-suppressed feeding test; TST, tail suspension test; BDNF, brain-derived neurotrophic factor; CREB, cAMP response element-binding protein; HPA, hypothalamic-pituitary-adrenal.

\section{Author contribution}

Yu-hua RAN and Xiao-xu HU contributed equally to this paper. Yu-hua RAN contributed to experiment design, data analysis, and manuscript writing. Xiao-xu HU performed the behavioral tests, and Yu-lu WANG performed the molecular chemistry tests. Nan ZHAO and Li-ming ZHANG aided with the in vitro behavioral tests and data analysis. Yun-feng LI and Hua-xia LIU contributed to the research design, data analysis, and manuscript revision.

\section{Acknowledgments}

This work is supported by the Beijing Municipal Natural Science Foundation № 7164290 .

\section{References}

1 Kessler RC, Berglund P, Demler O, Jin R, Koretz D, Merikangas KR, et al. The epidemiology of major depressive disorder: results from the National Comorbidity Survey Replication (NCS-R). JAMA 2003; 289: 3095-105.

2 Lee S, Tsang A, Zhang MY, Huang YQ, He YL, Liu ZR, et al. Lifetime prevalence and inter-cohort variation in DSM-IV disorders in metropolitan China. Psychol Med 2007; 37: 61-71.

3 Shen YC, Zhang MY, Huang YQ, He YL, Liu ZR, Cheng H, et al. Twelvemonth prevalence, severity, and unmet need for treatment of mental disorders in metropolitan China. Psychol Med 2006; 36: 257-67.

4 Trivedi MH, Rush AJ, Wisniewski SR, Nierenberg AA, Warden D, Ritz L, et al. Evaluation of outcomes with citalopram for depression using measurement-based care in STAR*D: implications for clinical practice. Am J Psychiatry 2006; 163: 28-40.

5 Penn E, Tracy DK. The drugs don't work? antidepressants and the current and future pharmacological management of depression. Ther Adv Psychopharmacol 2012; 2: 179-88.

6 Nutt DJ. The neuropharmacology of serotonin and noradrenaline in depression. Int Clin Psychopharmacol 2002; 17 Suppl 1: S1-12.

7 Shelton RC, Tomarken AJ. Can recovery from depression be achieved? Psychiatr Serv 2001; 52: 1469-78.

8 Khan A. Vilazodone, a novel dual-acting serotonergic antidepressant for managing major depression. Expert Opin Investig Drugs 2009; 18 : 1753-64.

9 Citrome L. Vilazodone for major depressive disorder: a systematic review of the efficacy and safety profile for this newly approved antidepressant - what is the number needed to treat, number needed to harm and likelihood to be helped or harmed? Int J Clin Practice 2012; 66: 356-68.

10 Reed CR, Kajdasz DK, Whalen H, Athanasiou MC, Gallipoli S, Thase ME. The efficacy profile of vilazodone, a novel antidepressant for the treatment of major depressive disorder. Cur Med Res Opin 2012; 28: 27-39.

11 Portella MJ, de Diego-Adelino J, Puigdemont D, Perez-Egea R, Alvarez E, Artigas $\mathrm{F}$, et al. Pindolol augmentation enhances response outcomes in first depressive episodes. Eur Neuropsychopharmacol 2009; 19: 516-9.

12 Sim-Selley LJ, Vogt LJ, Xiao R, Childers SR, Selley DE. Region-specific changes in 5- $\mathrm{HT}_{1 \mathrm{~A}}$ receptor-activated G-proteins in rat brain following chronic buspirone. Eur J Pharmacol 2000; 389: 147-53.

13 Czeh B, Perez-Cruz C, Fuchs E, Flugge G. Chronic stress-induced cellular changes in the medial prefrontal cortex and their potential clinical implications: does hemisphere location matter? Behavioural Brain Res 2008; 190: 1-13. 
14 Trivedi MH, Fava M, Wisniewski SR, Thase ME, Quitkin F, Warden D, et al. Medication augmentation after the failure of SSRIs for depression. N Engl J Med 2006; 354: 1243-52.

15 Banasr M, Duman RS. Glial loss in the prefrontal cortex is sufficient to induce depressive-like behaviors. Biol Psychiatr 2008; 64: 863-70.

16 Willner P, Towell A, Sampson D, Sophokleous S, Muscat R. Reduction of sucrose preference by chronic unpredictable mild stress, and its restoration by a tricyclic antidepressant. Psychopharmacology (Berl). 1987; 93: 358-64.

17 Gray JA, Lalljee B. Sex differences in emotional behaviour in the rat: correlation between open-field defecation and active avoidance. Animal Behav 1974; 22: 856-61.

18 Chen HX, Jin ZL, Zhang LM, Xue R, Xu XD, Zhao N, et al. Antidepressant-like activity of YL-0919: a novel combined selective serotonin reuptake inhibitor and $5-\mathrm{HT}_{1 \mathrm{~A}}$ receptor agonist. PLoS One 2013; 8: e83271.

19 Zhang LM, Wang XY, Zhao N, Wang YL, Hu XX, Ran YH, et al. Neurochemical and behavioural effects of hypidone hydrochloride ( $Y L-$ 0919): a novel combined selective 5-HT reuptake inhibitor and partial 5-HT1A agonist. Br J Pharmacol 2017; 174: 769-80.

20 Banasr M, Valentine GW, Li XY, Gourley SL, Taylor JR, Duman RS. Chronic unpredictable stress decreases cell proliferation in the cerebral cortex of the adult rat. Biol Psychiatry 2007; 62: 496-504.

21 Banasr M, Duman RS. Glial loss in the prefrontal cortex is sufficient to induce depressive-like behaviors. Biol Psychiatry 2008; 64: 863-70.

22 Ma M, Ren Q, Yang C, Zhang JC, Yao W, Dong C, et al. Adjunctive treatment of brexpiprazole with fluoxetine shows a rapid antidepressant effect in social defeat stress model: Role of BDNFTrkB signaling. Sci Rep 2016; 6: 39209.

23 Willner P, Towell A, Sampson D, Sophokleous S, Muscat R. Reduction of sucrose preference by chronic unpredictable mild stress, and its restoration by a tricyclic antidepressant. Psychopharmacology (Berl) 1987; 93: 358-64.

24 Gray JA, Lalljee B. Sex differences in emotional behaviour in the rat: correlation between open-field defecation and active avoidance. Anim Behav 1974; 22: 856-61.

25 Warner-Schmidt JL, Duman RS. VEGF is an essential mediator of the neurogenic and behavioral actions of antidepressants. Proc Natl Acad Sci U S A 2007; 104: 4647-52.

26 Masood A, Nadeem A, Mustafa SJ, O’Donnell JM. Reversal of oxidative stress-induced anxiety by inhibition of phosphodiesterase-2 in mice. J Pharmacol Exp Ther 2008; 326: 369-79.

27 Ren Q, Ma M, Ishima T, Morisseau C, Yang J, Wagner KM, et al. Gene deficiency and pharmacological inhibition of soluble epoxide hydrolase confers resilience to repeated social defeat stress. Proc Natl Acad Sci U S A 2016; 113: E1944-52.

28 Ren Q, Zhang JC, Ma M, Fujita Y, Wu J, Hashimoto K. 7,8-Dihydroxyflavone, a TrkB agonist, attenuates behavioral abnormalities and neurotoxicity in mice after administration of methamphetamine. Psychopharmacology 2014; 231: 159-66.

29 Berton O, McClung CA, Dileone RJ, Krishnan V, Renthal W, Russo SJ, et al. Essential role of BDNF in the mesolimbic dopamine pathway in social defeat stress. Science 2006; 311: 864-8.

30 Golden SA, Covington HE, 3rd, Berton O, Russo SJ. A standardized protocol for repeated social defeat stress in mice. Nat Protoc 2011; 6: 1183-91.

31 Qin X, Liu Y, Zhu M, Yang Z. The possible relationship between expressions of TRPC3/5 channels and cognitive changes in rat model of chronic unpredictable stress. Behav Brain Res 2015; 290: 180-6.

32 Flores G, Alquicer G, Silva-Gomez AB, Zaldivar G, Stewart J, Quirion $\mathrm{R}$, et al. Alterations in dendritic morphology of prefrontal cortical and nucleus accumbens neurons in post-pubertal rats after neonatal excitotoxic lesions of the ventral hippocampus. Neuroscience 2005; 133: 463-70.

33 Martinez-Tellez R, Gomez-Villalobos Mde J, Flores G. Alteration in dendritic morphology of cortical neurons in rats with diabetes mellitus induced by streptozotocin. Brain Res 2005; 1048: 108-15.

34 Sholl DA. Dendritic organization in the neurons of the visual and motor cortices of the cat. J Anat 1953; 87: 387-406.

35 Wang JW, David DJ, Monckton JE, Battaglia F, Hen R. Chronic fluoxetine stimulates maturation and synaptic plasticity of adult-born hippocampal granule cells. J Neurosci 2008; 28: 1374-84.

36 Papp M, Moryl E, Willner P. Pharmacological validation of the chronic mild stress model of depression. Eur J Pharmacol 1996; 296: 12936.

37 Willner P. Validity, reliability and utility of the chronic mild stress model of depression: a 10-year review and evaluation. Psychopharmacology 1997; 134: 319-29.

38 Sun JD, Liu Y, Yuan YH, Li J, Chen NH. Gap junction dysfunction in the prefrontal cortex induces depressive-like behaviors in rats. Neuropsychopharmacology 2012; 37: 1305-20.

39 Fawcett J, Clark DC, Scheftner WA, Gibbons RD. Assessing anhedonia in psychiatric patients. Arch Gen Psychiatry 1983; 40: 79-84.

40 McEwen BS. Glucocorticoids, depression, and mood disorders: structural remodeling in the brain. Metabolism 2005; 54: 20-3.

41 Willner P. Chronic mild stress (CMS) revisited: consistency and behavioural-neurobiological concordance in the effects of CMS. Neuropsychobiology 2005; 52: 90-110.

42 Zarate CA Jr, Singh JB, Carlson PJ, Brutsche NE, Ameli R, Luckenbaugh $\mathrm{DA}$, et al. A randomized trial of an $N$-methyl- $D$-aspartate antagonist in treatment-resistant major depression. Arch Gen Psychiatry 2006; 63: 856-64.

43 Berman RM, Cappiello A, Anand A, Oren DA, Heninger GR, Charney DS, et al. Antidepressant effects of ketamine in depressed patients. Biol Psychiatry 2000; 47: 351-4.

44 Liebrenz M, Borgeat A, Leisinger R, Stohler R. Intravenous ketamine therapy in a patient with a treatment-resistant major depression. Swiss Med Wkly 2007; 137: 234-6.

45 Bondi CO, Rodriguez G, Gould GG, Frazer A, Morilak DA. Chronic unpredictable stress induces a cognitive deficit and anxiety-like behavior in rats that is prevented by chronic antidepressant drug treatment. Neuropsychopharmacology 2008; 33: 320-31.

46 Crema L, Schlabitz M, Tagliari B, Cunha A, Simao F, Krolow R, et al. $\mathrm{Na}^{+}, \mathrm{K}^{+}$ATPase activity is reduced in amygdala of rats with chronic stress-induced anxiety-like behavior. Neurochem Res 2010; 35 : 1787-95.

47 Tafet GE, Bernardini R. Psychoneuroendocrinological links between chronic stress and depression. Prog Neuropsychopharmacol Biol Psychiatry 2003; 27: 893-903.

48 Morley-Fletcher S, Darnaudery M, Mocaer E, Froger N, Lanfumey L, Laviola G, et al. Chronic treatment with imipramine reverses immobility behaviour, hippocampal corticosteroid receptors and cortical 5- $\mathrm{HT}(1 \mathrm{~A})$ receptor $\mathrm{mRNA}$ in prenatally stressed rats. Neuropharmacology 2004; 47: 841-7.

49 Centeno VA, Volosin M. Chronic treatment with desipramine: effect on endocrine and behavioral responses induced by inescapable stress. Physiol Behav 1997; 62: 939-44.

50 Xu Y, Ku B, Tie L, Yao H, Jiang W, Ma X, et al. Curcumin reverses the effects of chronic stress on behavior, the HPA axis, BDNF expression and phosphorylation of CREB. Brain Res 2006; 1122: 56-64.

51 Budziszewska B. Effect of antidepressant drugs on the hypothalamicpituitary-adrenal axis activity and glucocorticoid receptor function. 
Pol J Pharmacol 2002; 54: 343-9.

52 Dias GP, Cavegn N, Nix A, do Nascimento Bevilaqua MC, Stangl $\mathrm{D}$, Zainuddin MS, et al. The role of dietary polyphenols on adult hippocampal neurogenesis: molecular mechanisms and behavioural effects on depression and anxiety. Oxid Med Cell Longev 2012; 2012: 541971.

53 Thome J, Sakai N, Shin K, Steffen C, Zhang YJ, Impey S, et al. cAMP response element-mediated gene transcription is upregulated by chronic antidepressant treatment. J Neurosci 2000; 20: 4030-6.

54 Akinfiresoye L, Tizabi Y. Antidepressant effects of AMPA and ketamine combination: role of hippocampal BDNF, synapsin, and mTOR. Psychopharmacology 2013; 230: 291-8.

55 Delgado PL. Common pathways of depression and pain. J Clin Psychiatry 2004; 65 Suppl 12: 16-9.
56 Serrats J, Artigas F, Mengod G, Cortes R. An autoradiographic study of the influence of pindolol upon $\left[{ }^{35} \mathrm{~S}\right] \mathrm{GTPgammaS}$ binding in rat, guinea pig and human brain. Int J Neuropsychopharmacol 2004; 7: 27-34.

57 Czeh B, Perez-Cruz C, Fuchs E, Flugge G. Chronic stress-induced cellular changes in the medial prefrontal cortex and their potential clinical implications: does hemisphere location matter? Behav Brain Res 2008; 190: 1-13.

58 Cao G, Harris KM. Developmental regulation of the late phase of longterm potentiation (L-LTP) and metaplasticity in hippocampal area CA1 of the rat. J Neurophysiol 2012; 107: 902-12.

59 Bourne JN, Harris KM. Coordination of size and number of excitatory and inhibitory synapses results in a balanced structural plasticity along mature hippocampal CA1 dendrites during LTP. Hippocampus 2011; 21: 354-73. 